\title{
Clinical significance of the phosphorylation of MAPK and protein expression of cyclin D1 in human osteosarcoma tissues
}

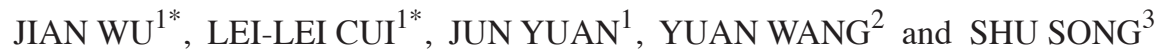 \\ ${ }^{1}$ Department of Laboratory Medicine, The First People's Hospital of Yancheng City, Yancheng, Jiangsu 224005; ${ }^{2}$ Department \\ of Laboratory Medicine, The First Affiliated Hospital of Zhejiang Chinese Medical University, Hangzhou, Zhejiang 310006; \\ ${ }^{3}$ Pathological Science Laboratory, The First People's Hospital of Yancheng City, Yancheng, Jiangsu 224005, P.R. China
}

Received January 13, 2016; Accepted January 4, 2017

DOI: $10.3892 / \mathrm{mmr} .2017 .6224$

\begin{abstract}
The aim of the present study was to investigate the significance of the phosphorylation of mitogen-activated protein kinase (MAPK) and the protein expression of cyclin D1 in human osteosarcoma tissues. Human osteosarcoma tissue samples were collected from 30 patients, benign bone tumor samples were collected from 30 patients, and normal bone tissues were collected from 10 individuals as controls. Immunohistochemistry was performed to measure the levels of phosphorylated (p)-MAPK and cyclin D1 protein in cases of human osteosarcoma. The results showed that the positive rates of MAPK and cyclin D1 in osteosarcoma were $86.67 \%$ (26/30) and $73.00 \%$ (22/30), respectively. The positive staining rates of MAPK and cyclin D1 in benign bone tumor tissues were $10.00 \%(3 / 30)$ and $3.30 \%(1 / 30)$, respectively. The positive rate in the normal bone tissues was $0 \%(0 / 30)$, which was significantly lower, compared with that of the cancerous bone tissue. The positive rates of MAPK and cyclin D1 in osteosarcoma were increased $(\mathrm{P}<0.05)$, and the expression of cyclin D1 and p-MAPK were positively correlated. The phosphorylation of MAPK may be important in the development of osteosarcoma, and the overactivation of MAPK may induce high expression of cyclin D1 and induce tumor cells to proliferate continuously.
\end{abstract}

Correspondence to: Dr Yuan Wang, Department of Laboratory Medicine, The First Affiliated Hospital of Zhejiang Chinese Medical University, 54 Youdian Road, Hangzhou, Zhejiang 310006, P.R. China

E-mail: piaoxue1982717@sina.com

Dr Shu Song, Pathological Science Laboratory, The First People's Hospital of Yancheng City, 16 Yuehe Road, Yancheng, Jiangsu 224005, P.R. China

E-mail: 2295422684@qq.com

${ }^{*}$ Contributed equally

Key words: human osteosarcoma, mitogen-activated protein kinase, cyclin D1, phosphorylation

\section{Introduction}

Osteosarcoma is the most common primary tumor of bone tissue, is more common in young individuals and exhibits a high degree of malignancy $(1,2)$. The treatment of osteosarcoma comprises neoadjuvant chemotherapy and surgery. The five-year survival rate remains at a level of 60-70\%, and has not increased significantly (3). The primary reason for this is that the mechanism underlying its pathogenesis remains to be fully elucidated. The formation and development of the tumor is multifactorial, multistage and a gradual evolution process. Early diagnosis and timely intervention is essential for improving the prognosis. Investigation of the association between cell proliferation and cell signaling has become an area of increased interest $(4,5)$.

As the axis of several types of signal channels, mitogen-activated protein kinase (MAPK) cascade activation is a key member in the receipt of signals, which are transferred and carried by membrane receptors into the nucleus $(6,7)$, which is key to numerous signaling channels associated with cell proliferation.

The overexpression of cyclin D1, which is referred to as one of the regulatory factors in the cell cycle, is a characteristic of several types of human primary tumor, and is of vital significance for the diagnoses and prognoses of tumors $(8,9)$.

In order to elucidate molecular abnormalities of the signaling cascade in the growth inhibition and growth of osteosarcoma, cell proliferation and malignant transformation-associated mechanisms, the present study detected MAPK, phosphorylated (p)-MAPK and cyclin D1 using immunohistochemical staining in tissues of osteosarcoma and benign bone tumors, and in normal bone tissues as a control. The association between p-MAPK and expression of cyclin D1, and the mechanism underlying the formation of osteosarcoma were examined to identify novel techniques for its early diagnosis.

\section{Materials and methods}

Patients and controls. In the last three years, samples of human osteosarcoma and benign bone tumor tissues were collected from 60 patients (30 patients with human osteosarcoma and 30 patients with benign bone tumor), who received surgical 
Table I. EnVision immunohistochemical staining for p-MAPK in human osteosarcoma, benign bone tumor and normal bone tissues.

\begin{tabular}{|c|c|c|c|c|c|c|c|}
\hline \multirow[b]{2}{*}{ Group } & \multirow[b]{2}{*}{$\mathrm{n}$} & \multicolumn{3}{|c|}{ p-MAPK positive } & \multirow[b]{2}{*}{ p-MAPK negative } & \multirow[b]{2}{*}{ Positive rate $(\%)$} & \multirow[b]{2}{*}{ Strong positive rate $(\%)$} \\
\hline & & + & $2+$ & $3+$ & & & \\
\hline Normal bone tissue & 10 & 0 & 0 & 0 & 10 & 0 & 0 \\
\hline Benign bone tumor & 30 & 1 & 1 & 1 & 27 & 10.00 & 3.33 \\
\hline Human osteosarcoma & 30 & 4 & 10 & 12 & 4 & 86.67 & 40.00 \\
\hline
\end{tabular}

The $\chi^{2}$ test was used to compare positive staining between human osteosarcoma and normal bone tissues $(\mathrm{P}<0.001)$, between benign bone tumor and human osteosarcoma $(\mathrm{P}=0.09)$ and between benign bone tumor and normal bone tissues $(\mathrm{P}=0.014)$. + , low expression; $2+$, moderate expression; 3+, high expression; p-MAPK, phosphorylated mitogen-activated protein kinase.

Table II. EnVision immunohistochemical staining for cyclin D1 in human osteosarcoma, benign bone tumor and normal bone tissues.

\begin{tabular}{|c|c|c|c|c|c|c|c|}
\hline \multirow[b]{2}{*}{ Group } & \multirow[b]{2}{*}{$\mathrm{n}$} & \multicolumn{3}{|c|}{$\begin{array}{l}\text { Cyclin D1 } \\
\text { positive }\end{array}$} & \multirow[b]{2}{*}{ Cyclin D1 negative } & \multirow[b]{2}{*}{ Positive rate $(\%)$} & \multirow[b]{2}{*}{ Strong positive rate $(\%)$} \\
\hline & & + & $2+$ & $3+$ & & & \\
\hline Normal bone tissues & 10 & 0 & 0 & 0 & 10 & 0 & 0 \\
\hline Benign bone tumor & 30 & 0 & 1 & 0 & 29 & 3.30 & 0.00 \\
\hline Human osteosarcoma & 30 & 7 & 5 & 10 & 8 & 73.00 & 33.33 \\
\hline
\end{tabular}

The $\chi^{2}$ test was used to compare positive staining between human osteosarcoma and normal bone tissues $(\mathrm{P}<0.001)$, between benign bone tumor and human osteosarcoma tissues $(\mathrm{P}=0.006)$ and between benign bone tumor and normal bone tissues $(\mathrm{P}=0.125)$.,+ low expression; $2+$, moderate expression; $3+$, high expression.

resection at The First People's Hospital of Yancheng City (Yancheng, China), and had been diagnosed via pathological confirmation. Detailed clinical and pathological data were collected from each patient, and none of the patients had received preoperative chemotherapy or radiotherapy. The patients with osteosarcoma included 20 men and 10 women, aged between 25 and 73 years (mean $40.9 \pm 11.6$ years). The benign bone tumor patient group included 19 men and 11 women, aged between 40 and 73 years (mean $54.8 \pm 12.2$ years). Normal bone tissue specimens were collected by surgical resection from 10 individuals to serve as a control group. These included five men and five women, aged between 36 and 68 years (mean $49.4 \pm 10.3$ years). No statistically significant differences were detected in age or gender among the three groups. All specimens were obtained following the provision of informed patient consent and were approved by the Ethics Committee of The First People's Hospital of Yancheng City [ID no. HMU (Ethics) 20121103].

Immunohistochemical staining techniques. The EnVision immunohistochemical staining method was used to detect the distributions of p-MAPK and cyclin D1. The immunohistochemical procedures were performed in strict accordance with the manufacturer's protocols. The EnVision and DAB chromogenic reagent kits (Antibody Diagnostic, Inc., New York, NY, USA) were used for immunohistochemical staining. All staining was performed under the same conditions, in which the tissue specimen was sliced into $4 \mu \mathrm{m}$ sections, dehydrated and dewaxed, and antigen retrieval was performed using $0.01 \mathrm{~mol} / 1$ citric acid ( $\mathrm{pH}$ 6.0). Normal goat serum (Toyobo Co., Ltd., Osaka, Japan) was added to the tissue sections and incubated for $10 \mathrm{~min}$ at room temperature, following which the corresponding specific antibody (cat. no. 7074; Cell Signaling Technology, Inc., Danvers, MA, USA; dilution, 1:1,000) were added to the tissue section and incubated for $1.5 \mathrm{~h}$ at room temperature. The sections were washed three times with PBS for $3 \mathrm{~min}$. The secondary antibody (cat. no. 4370; Cell Signaling Technology, Inc.; dilution, 1:1,000) was added and incubated for $30 \mathrm{~min}$ at room temperature. Staining was performed using DAB and nuclei were stained using hematoxylin. The tissue sections were then dehydrated in gradient ethanol, cleared using xylene and sealed using natural gum. Each group stained had a positive control, with the known positive section reagent provided by the reagent company (p-MAPK; cat. no. 9216; Cell Signaling Technology, Inc.; dilution, 1:1,000) (10), and a negative control, in which the corresponding specific antibody was replaced with PBS.

Staining of the nucleus in yellow or tan reactant particles indicated positivity. The staining was examined in four independent experiments for random detection using an Olympus optical microscope (BH-2; Olympus Corporation, Tokyo, Japan) at high magnification (magnification, x200). According 
A
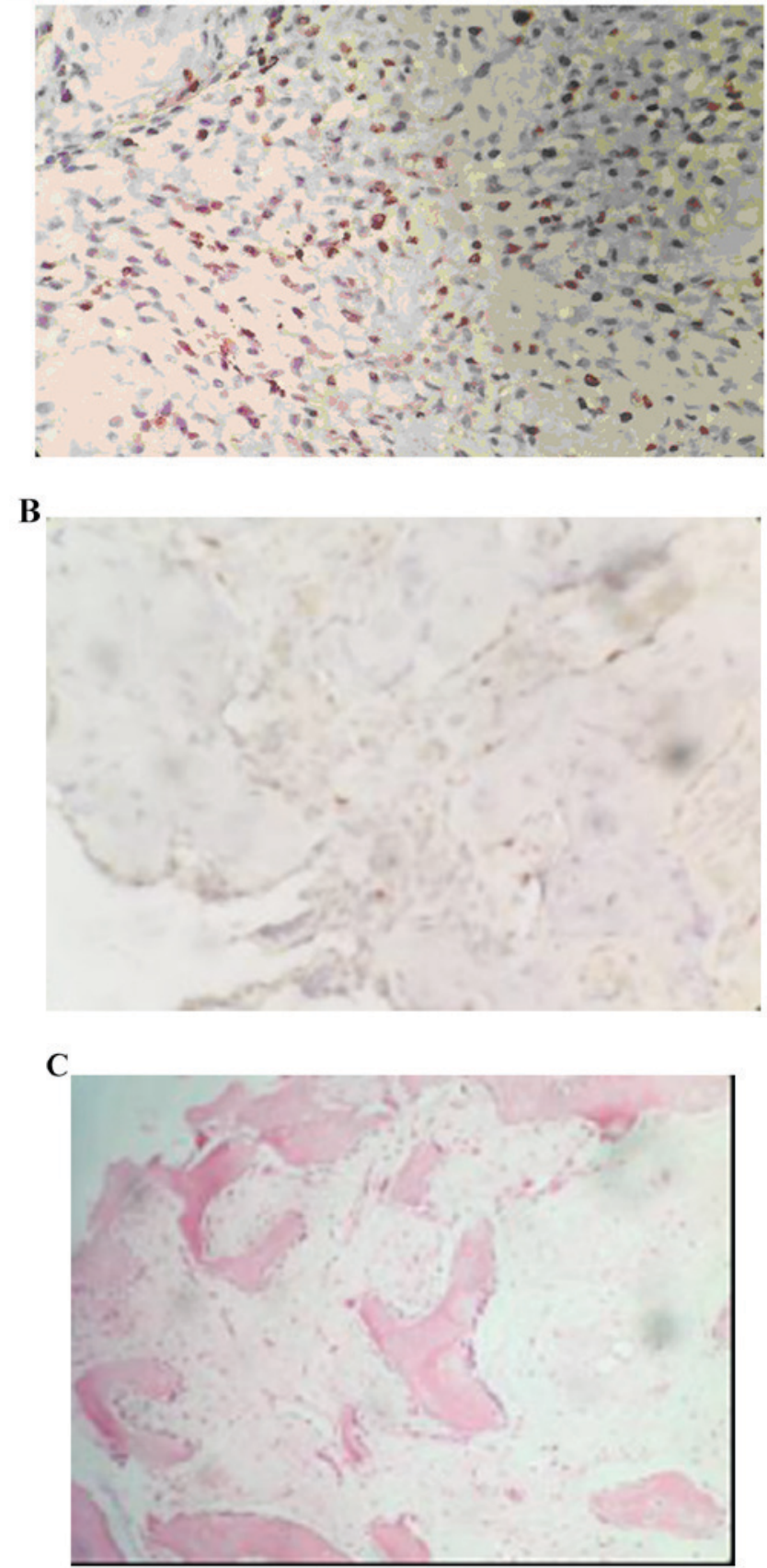

Figure 1. Staining using EnVision immunohistochemistry for p-MAPK in human osteosarcoma, benign bone tumor and normal bone tissues. (A) Positive staining for p-MAPK in human osteosarcoma; (B) weakly positive staining for p-MAPK in benign bone tumor tissues; (C) negative staining for p-MAPK in normal bone tissues (magnification, x200). p-MAPK, mitogen-activated protein kinase.

to the degree of positive staining and the percentage of tumor cells, the criteria for assessment were as follows: Negative expression (-), marginal cell shading, expression of $<5 \%$; low expression (+), pale yellow or positive staining of 5-29\%; moderate expression $(2+)$, yellow or positive staining of 30-59\%; high expression (3+), tan colored or positive staining of $>60 \%$.

Statistical analysis. SPSS 13.0 statistical software (SPSS, Inc., Chicago, IL, USA) was used for statistical analysis. The $\chi^{2}$ test was used to compare the distribution of p-MAPK and cyclin D1 among osteosarcoma tissues, benign bone tumor tissues and normal bone tissues, and Spearman's correlation was used to analyze the association between the distribution of p-MAPK and cyclin D1. P $<0.05$ was considered to indicate a statistically significant difference.

\section{Results}

Distribution of nuclear staining of p-MAPK in human osteosarcoma, benign bone tumor and normal bone tissues. The positive rate of p-MAPK staining in the human osteosarcoma tissues was $86.67 \%$ (26/30). The positive rate of p-MAPK in the benign bone tumor tissues was $10.00 \%$ (3/30), and, staining was significantly higher, compared with that in the normal bone tissue $(0 ; \mathrm{P}<0.05)$. The staining intensity of $\mathrm{p}$-MAPK in the human osteosarcoma tissues was significantly higher, compared with the staining intensity of p-MAPK in the benign bone tumor tissues $(\mathrm{P}<0.05$; Fig. 1A-C; Table I).

Cell nuclear staining distribution of cyclin D1 in human osteosarcoma, benign bone tumor and normal bone tissues. The positive rate of cyclin D1 staining in the human osteosarcoma tissues was $73.00 \%(22 / 30)$. The positive rate of cyclin D1 in the benign bone tumor was $3.30 \%(1 / 30)$, and staining was negative in normal bone tissues. The staining intensity of cyclin D1 in the benign bone tumor was significantly higher, compared with that in normal bone tissue $(0 ; \mathrm{P}<0.05)$. The staining intensity of cyclin D1 in the human osteosarcoma tissues was significantly higher, compared with the staining intensity of cyclin $\mathrm{D} 1$ in the benign bone tumor tissues $(\mathrm{P}<0.05$; Fig. 2A-C; Table II).

Correlation between $p-M A P K$ and cyclin D1 in human osteosarcoma. To analyze the mutual associations among the proteins according to the expression of each antigen in human osteosarcoma, Spearman's correlation coefficient analysis was used. p-MAPK and cyclin D1 were positively associated with the intensity of positive staining ( $\mathrm{r}=0.714$; $\mathrm{P}<0.05$; Table III).

\section{Discussion}

The occurrence and development of cancer is closely associated with abnormalities in the transfer and regulation of cellular signaling $(11,12)$. Several signaling channels of carcinogenic tyrosine kinase have a convergence point with MAPK. MAPK remains static in unstimulated cells, however, it is activated following the receipt of activation signals from MAPK kinase (MKK) and MKK kinase (13), and is phosphorylated in a stepwise manner in cells stimulated by growth factors (14). When it is activated, MAPK transfers into the nucleus and activates certain oncogenes to stimulate the proliferation of cells and inhibit apoptosis.

The results of the present study showed that the positive rates of MAPK staining in osteosarcoma and benign bone tumors were higher, compared with that in normal bone tissues, and the expression of p-MAPK was significantly higher, compared with that in normal bone tissues. These results suggested that activation of the MAPK cascade is important 
Table III. Staining of p-MAPK and cyclin D1 in human osteosarcoma cases.

\begin{tabular}{|c|c|c|}
\hline \multirow[b]{2}{*}{$\mathrm{n}$} & \multicolumn{2}{|c|}{ Positive staining rate (\%) } \\
\hline & p-MAPK & Cyclin D1 \\
\hline 1 & 5 & 5 \\
\hline 2 & 8 & 5 \\
\hline 3 & 10 & 2 \\
\hline 4 & 30 & 35 \\
\hline 5 & 12 & 20 \\
\hline 6 & 10 & 6 \\
\hline 7 & 15 & 8 \\
\hline 8 & 65 & 60 \\
\hline 9 & 4 & 3 \\
\hline 10 & 8 & 57 \\
\hline 11 & 7 & 5 \\
\hline 12 & 4 & 3 \\
\hline 13 & 5 & 3 \\
\hline 14 & 65 & 60 \\
\hline 15 & 8 & 30 \\
\hline 16 & 45 & 60 \\
\hline 17 & 35 & 30 \\
\hline 18 & 8 & 3 \\
\hline 19 & 3 & 4 \\
\hline 20 & 5 & 5 \\
\hline 21 & 75 & 15 \\
\hline 22 & 2 & 4 \\
\hline 23 & 90 & 50 \\
\hline 24 & 30 & 15 \\
\hline 25 & 10 & 10 \\
\hline 26 & 10 & 8 \\
\hline 27 & 70 & 55 \\
\hline 28 & 5 & 1 \\
\hline 29 & 12 & 30 \\
\hline 30 & 28 & 6 \\
\hline
\end{tabular}

p-MAPK and cyclin D1 ( $\mathrm{r}=0.714 ; \mathrm{P}<0.05)$. p-MAPK, phosphorylated mitogen-activated protein kinase.

in the occurrence of osteosarcoma. In addition, the degree of malignant bone tumor tissue p-MAPK staining intensity was significantly higher, compared with that of benign tumor tissue. Therefore, the results suggested that the overactivation of MAPK may be closely associated with the invasive growth potential of osteosarcoma.

Studies have indicated that the overexpression of cyclin D1 may result in a reduction of the G1 phase of the cell cycle (15-17), leading to progression into the $S$ phase and completing the duplication of DNA. The increase in the protein expression of cyclin D1 is observed in certain primary malignant tumors, including parathyroid adenoma, neck squamous-cell carcinoma, breast cancer, esophageal cancer, and hepatocellular carcinoma (18-21). As MAPK is expressed as an upstream gene of cyclin D1, the increase in the level of
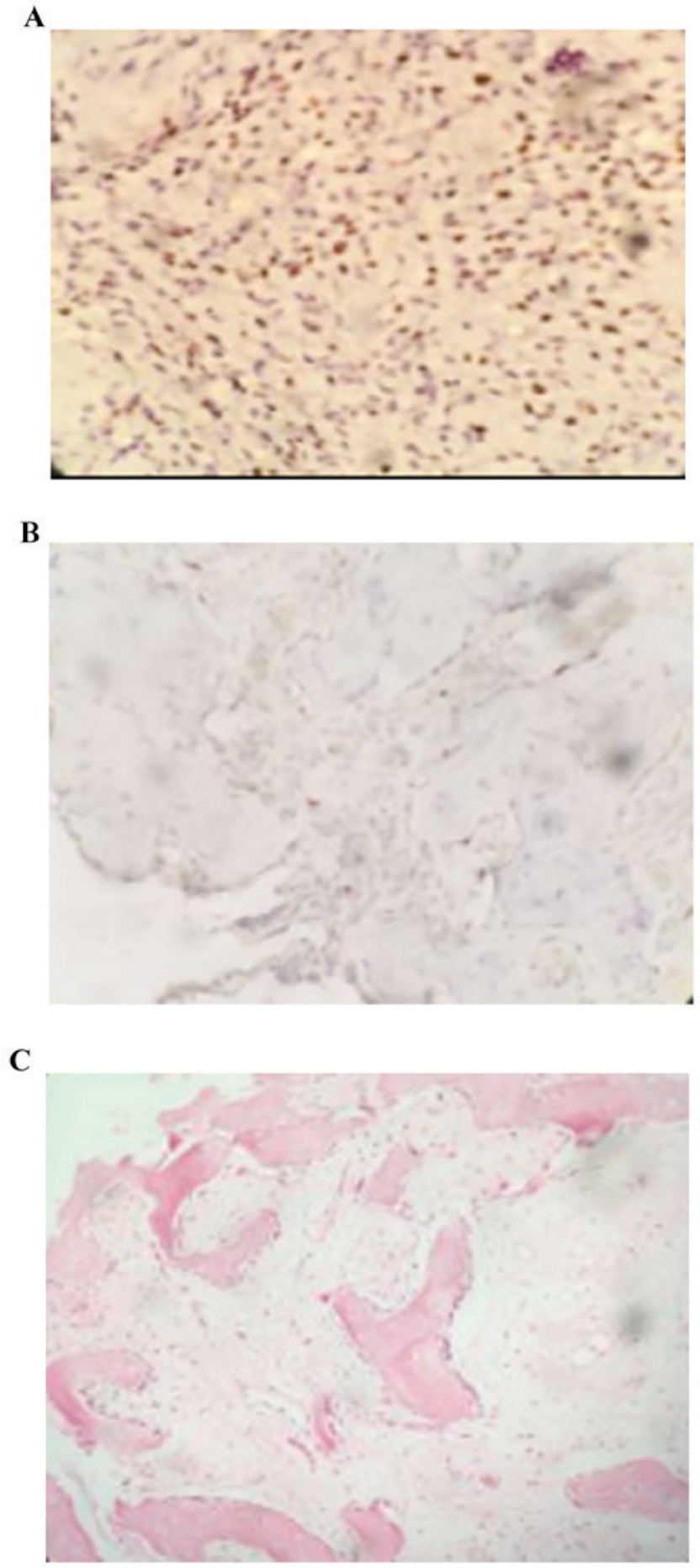

Figure 2. Staining using EnVision immunohistochemistry for cyclin D1 in human osteosarcoma, benign bone tumor and normal bone tissues. (A) Positive staining for cyclin D1 in human osteosarcoma; (B) weakly positive staining for cyclin D1 in benign bone tumor tissue; (C) negative staining for cyclin D1 in normal bone tissues (magnification, x200).

p-MAPK in osteosarcoma was directly proportional to the intensity of cyclin D1-positive staining.

The results of the present study also demonstrated that the positive rate of cyclin D1 staining was significantly higher in the osteosarcoma tissues, compared with rates in normal bone tissues and benign bone tumors. In addition, there was positive correlation between the two genes in osteosarcoma, determined by cyclin D1 and p-MAPK positive intensity. MAPK 
is expressed upstream of cyclin D1, and overexpression of the cyclin D1 may be induced by the p-MAPK signaling pathway in osteosarcoma, which can lead to increased proliferation of the tumor cells.

In conclusion, the present study demonstrated that the expression of p-MAPK and cyclin D1 were suitable for use as markers for osteosarcoma to assist in early diagnosis and prognosis. This was achieved by examining the expression levels of p-MAPK and cyclin D1 in osteosarcoma, benign bone tumor tissues and normal bone tissues, and examining the association between the two. By interfering with the p-MAPK signal transduction pathway in human osteosarcoma, it is possible to prevent the overexpression of cyclin D1 in the tissue, which may assist in preventing the occurrence of osteosarcoma. This provides a novel technique and offers potential for clinical use in the treatment of osteosarcoma.

\section{References}

1. Huang J, Liu K, Song D, Ding M, Wang J, Jin Q and Ni J: Krüppel-like factor 4 promotes high-mobility group box 1 -induced chemotherapy resistance in osteosarcoma cells. Cancer Sci 107: 242-249, 2016.

2. Li YS, Deng ZH, Zeng C and Lei GH: JNK pathway in osteosarcoma: Pathogenesis and therapeutics. J Recept Signal Transduct Res 36: 465-470, 2016.

3. Chakravarthi PS, Kattimani VS, Prasad LK and Satish PR: Juxtacortical osteosarcoma of the mandible: Challenges in diagnosis and management. Natl J Maxillofac Surg 6: 127-131, 2015.

4. Zhou W, Zhu Y, Chen S, Xu R and Wang K: Fibroblast growth factor receptor 1 promotes MG63 cell proliferation and is associated with increased expression of cyclin-dependent kinase 1 in osteosarcoma. Mol Med Rep 13: 713-719, 2016.

5. Yang J, Cheng D, Zhou S, Zhu B, Hu T and Yang Q: Overexpression of X-Box Binding Protein 1 (XBP1) Correlates to Poor Prognosis and Up-Regulation of PI3K/mTOR in Human Osteosarcoma. Int J Mol Sci 16: 28635-28646, 2015.

6. Shao Y, Wang C, Hong Z and Chen Y: Inhibition of p38 mitogen-activated protein kinase signaling reduces multidrug transporter activity and anti-epileptic drug resistance in refractory epileptic rats. J Neurochem 136: 1096-1105, 2016.

7. Zhang J, Liu Q, Fang Z, Hu X, Huang F, Tang L and Zhou S: Hypoxia induces the proliferation of endothelial progenitor cells via upregulation of Apelin/APLNR/MAPK signaling. Mol Med Rep 13: 1801-1806, 2016.

8. Xu W, Yang Z, Zhou SF and Lu N: Posttranslational regulation of phosphatase and tensin homolog (PTEN) and its functional impact on cancer behaviors. Drug Des Devel Ther 8 : $1745-1751,2014$
9. Liang N, Zhang C, Dill P, Panasyuk G, Pion D, Koka V, Gallazzini M, Olson EN, Lam H, Henske EP, et al: Regulation of YAP by mTOR and autophagy reveals a therapeutic target of tuberous sclerosis complex. J Exp Med 211: 2249-2263, 2014.

10. Wu J, Lu WY and Cui LL: Clinical significance of STAT3 and MARK Phosphorylation, and the protein expression of cyclin D1 in skin squamous cell carcinoma tissues. Mol Med Rep 12: 8129-8134, 2015

11. Talbot JJ, Song X, Wang X, Rinschen MM, Doerr N, LaRiviere WB, Schermer B, Pei YP, Torres VEand Weimbs T: The cleaved cytoplasmic tail of polycystin-1 regulates Src-dependent STAT3 activation. J Am Soc Nephrol 25: 1737-1748, 2014.

12. Zhang YH, Li B, Shen L, Shen Y and Chen XD: The role and clinical significance of YES-associated protein 1 in human osteosarcoma. Int J Immunopathol Pharmacol 26: 157-167, 2013

13. Zheng ZP, Yan Y, Xia J, Zhang S, Wang M, Chen J and Xu Y: A phenylacetaldehyde-flavonoid adduct, 8-C-(E-phenylethenyl)norartocarpetin, exhibits intrinsic apoptosis andMAPK pathways-related anticancer potential on HepG2, SMMC-7721 and QGY-7703. Food Chem 197: 1085-1092, 2016.

14. Cursons J, Angel CE, Hurley DG, Print CG, Dunbar PR, Jacobs MD and Crampin EJ: Spatially transformed fluorescence image data for ERK-MAPK and selected proteins within human epidermis. Gigascience 4: 63, 2015.

15. Beauvais S, Drevelle O, Lauzon MA, Daviau A and Faucheux N: Modulation of MAPK signalling by immobilized adhesive peptides: Effect on stem cell response to BMP-9-derived peptides. Acta Biomater 31: 241-251, 2016.

16. Dreyer JH, Hauck F, Barros MH and Niedobitek G: pRb and CyclinD1 Complement p16 as Immunohistochemical Surrogate Markers of HPV Infection in Head and Neck Cancer. Appl Immunohistochem Mol Morphol 2015 (Epub ahead of print).

17. Wu J, Lv S, An J and Lu C: Pre-miR-149 rs71428439 polymorphism is associated with increased cancer risk and AKT1/ cyclinD1 signaling in hepatocellular carcinoma. Int J Clin Exp Med 8: 13628-13633, 2015.

18. Yang Y, Zhao LH, Huang B, Wang RY, Yuan SX, Tao QF, Xu Y, Sun HY, Lin $\mathrm{C}$ and Zhou WP: Pioglitazone, a PPAR $\gamma$ agonist, inhibits growth and invasion of human hepatocellular carcinoma via blockade of the rage signaling. Mol Carcinog 54: 1584-1595, 2015.

19. Shi QQ, Zuo GW, Feng ZQ, Zhao LC, Luo L, You ZM, Li DY, Xia J, Li J and Chen DL: Effect of trichostatin a on anti HepG2 liver carcinoma cells: Inhibition of HDAC activity and activation of Wnt/ $\beta$-catenin signaling. Asian Pac J Cancer Prev 15: 7849-7855, 2014

20. Wang X, Liu H, Wang X, Zeng Z, Xie LQ, Sun ZG and Wei MX: Preventive effect of Actinidia valvata Dunn extract on N-methyl-N'-nitro-N-nitrosoguanidine-induced gastrointestinal cancer in rats. Asian Pac J Cancer Prev 15: 6363-6377, 2014.

21. Gopalakrishnan N, Saravanakumar M, Madankumar P, Thiyagu $\mathrm{M}$ and Devaraj $\mathrm{H}$ : Colocalization of $\beta$-catenin with Notch intracellular domain in colon cancer: A possible role of Notch1 signaling in activation of CyclinD1-mediated cell proliferation. Mol Cell Biochem 396: 281-293, 2014. 